\title{
A Distributed Coordination Framework for On-line Scheduling and Power Demand Balancing of Households Communities
}

\author{
Rodrigo Verschae, Hiroaki Kawashima, Takekazu Kato and Takashi Matsuyama
}

\begin{abstract}
The development of energy management systems (EMS) has attracted increasing attention during the last years. One of the main goals of EMS is to balance the power usage and generation, while also maintaining the quality of life (QoL) of the users. In this paper, a distributed coordination framework for on-line scheduling of appliances and control of the aggregated power demand of households is proposed. Each household consists of a set of appliances, and each appliance is modeled using a probabilistic generative model of its power usage profile, which can be updated through the day to indicate changes in user preferences. The coordination framework is formulated as a receding horizon distributed optimization, where the households' QoL, and the deviation from a scheduled usage are taken into account. The implemented distributed optimization can be seen as a negotiation among the households and a coordinator: the coordinator seeks to balance the aggregated power consumption by minimizing the deviation from a scheduled aggregated power usage, while each household tries not to deviate much from its preferred usage pattern.
\end{abstract}

\section{INTRODUCTION}

Power grid management systems are evolving towards a Internet-like network of distributed devices that produce / consume energy and that can exchange information about their energy use. This is allowing to control these networked devices in a coordinated way, and thus a more effective management of the available resources. The increasing need for energy efficiency, together with environmental issues, and the deployment of advanced metering infrastructure is driving the design of these energy management systems (EMS). Within this context, we are interested in the development of a demand-side management system where: $i$ ) the households in a community coordinate their power usage, and ii) control is not enforced on the users but the schedule is coordinated taking into account their Quality of Life (QoL).

Several demand-side management systems exist [1], [2], [3], going from approaches where the utility applies direct load control or uses of dynamic pricing to drive power usage [4], [5], [6], to recently proposed methods that seek to be consumer-friendly [7], [8], [9], [10], [11] by taking QoL into account. The scheduling of electric loads that can be deferred, such as the charging of electric vehicles (EV) [12], [13], together with the control of thermostatically controlled loads [14], [8], such as air conditioning, are some of the enablers and drivers of the development of demand management systems. Continuing the line of research proposed in

This work was supported by CREST from the Japan Science and Technology.

*Graduate School of Informatics, Kyoto University, Kyoto 6068501, Japan. Emails: rodrigo@verschae.org, kawashima@i.kyoto-u.ac.jp, tkato@vision.kuee.kyoto-u.ac.jp, tm@i.kyoto-u.ac.jp.
[11], we use a distributed coordination method for balancing the aggregated power of a community of households ${ }^{1}$ that takes into account the QoL of each household.

Coordinated and distributed power balancing is growing in importance because power balancing is directly related to the cost and stability of the power grid. In particular, the steadily increase of devices such as EV is requiring and enabling a coordinated scheduling [15], [13], [12]. This can help ensure stability by reducing peaks and valleys, thus addressing the supply-demand balance by smoothing the demand and responding to frequency variations. The stability issue has been traditionally managed by ancillary services (in a reactive way), thought it can be addressed more effectively when the demand-side contributes to it (see e.g. [16], [17]). The distributed control of power resources, which has been studied in the utility side [18], [19], [17] and in the demand side [15], can reduce the need to pass information about local cost functions [20] usually determined by a large amounts of (private) data. In our context this private data consists of daily appliance power usage patterns.

We propose the on-line distributed coordination of power usage within a community of households, with the main goal of balancing the power usage profile but also taking into account QoL and privacy. Our scenario is close to [21], which tries to minimize the deviation from a day-ahead power usage schedule and also takes into account the households' QoL. In order to maintain the QoL and to effectively schedule the appliances, modeling the flexibility of appliance usage is of key importance. While the modeling of flexibility has been discussed in the literature (e.g., [5], [22], [23], [10]), we utilize a generative probabilistic model, which can be learned from data of daily usage patterns, to describe the QoL through the flexibility of appliances. This model can describe controllable and non controllable appliances and can be updated through the day (similarly to [24]), thus taking into account load uncertainty and usage information.

Our work builds up on the ideas proposed in recent work [11], where a coordinated scheduling for balancing the power consumptions in households by exploiting a distributed algorithm called the alternating direction method of multipliers (ADMM) [25] and a probabilistic mode-switching model (which will be described in Section II) was developed. In particular, ADMM is now attracting great attention of the control community (e.g., [26], [20], [27], [28], [29]) for its fast and robust convergence in real problems. In particular,

\footnotetext{
${ }^{1} \mathrm{~A}$ household is understood as any group of appliances that can be controlled (not only at homes), such as offices, buildings, etc.
} 
it has been used in distributed electric vehicle charging control [30] [31] and optimal power flow [32] [33] [25]. The main contribution of this paper is to extend the scheme introduced in [11] to an on-line scheduling problem and to show in simulation how effective the proposed ADMMbased receding horizon optimization is for distributed powerbalancing problems. In particular, we show how the proposed method responds to changes in the households' behavior, modeled as changes in the probabilistic generative model.

The paper is structured as follows. We first give an overview of the framework (Section II), and afterwards we continue with a detailed formulation (Section III), where we describe the appliance and household models, the coordinator role, the receding horizon formulation, the distributed optimization and negotiation, and the optimal mode scheduling of the appliances. Later we present simulation results (Section IV) to finally conclude (Section V).

\section{FRAMEWORK}

The purpose of the proposed framework (see Fig. 1) is to implement a system to coordinate the power usage of a community of households, that takes into account the QoL. The coordination of the households is done by the community itself, instead of being controlled directly from the utility company. The community has a coordinator, which together with the households seek to balance the aggregated power usage of the community through the day by following a dayahead coordinated plan. In the same way, each household has an EMS that manages the devices at the household. (In the following we will use "household" to refer to the household's EMS when clear from context). It is assumed that the households have some degree of flexibility in their appliance usage, e.g. by controlling the power usage level or scheduling some appliances.

The coordinator provides support, having two main goals: to negotiate, with all households, a day-ahead schedule of the community's aggregated power usage, and to perform an on-line negotiation for adjusting to deviations from the plan that may occur through the day ${ }^{2}$.

The proposed framework makes a few assumptions, all of which have been already applied in real-world scenarios: $i$ ) the household can measure the power usage of its appliances, ii) the household can control or schedule some of the appliances, and iii) in the case when the appliance can not be controlled, the appliance (or the user) may inform the household its most likely schedule and power usage.

\section{FORMULATION}

\section{A. Appliances and Household}

Each household $i=1, \ldots, N$ consists of a set of $N_{i}$ appliances, $a_{i}=1, \ldots, N_{i}$, where appliance $a_{i}$ has a power consumption profile $x_{i, a_{i}} \in \mathbb{R}^{T}$, with $T$ the number of time-slots within a time period (e.g. a day). For example

\footnotetext{
${ }^{2} \mathrm{We}$ assume that the community buys energy from the utility and that the energy cost is related to the flatness of the power usage of the community, however we do not analyze how this cost may be split among the households and instead we focus on the coordinated scheduling of the appliances.
}

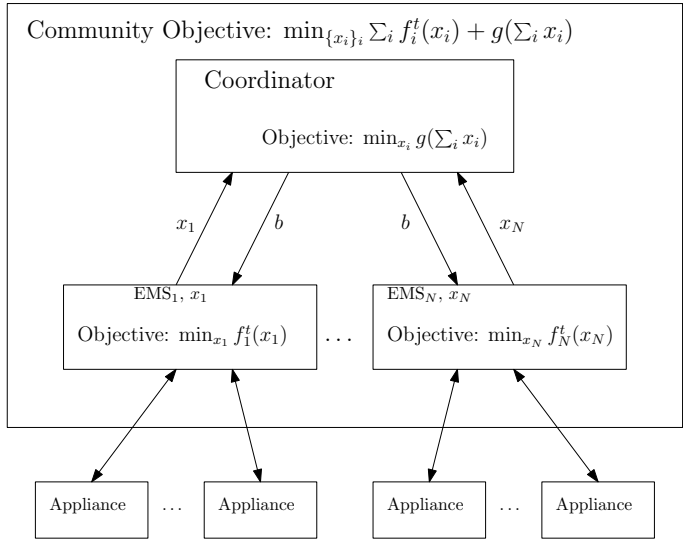

Fig. 1. System diagram. At each time step $t$, an iterative negotiation takes places where the coordinator seeks to balance the aggregated power usage profile, as measured by $\sum_{i} x_{i}$, and each household seeks to minimize the loss in QoL associated to its power usage profile $x_{i}$, as measured by $f_{i}^{t}\left(x_{i}\right)$.

the power usage profile for one day can be represented with $T=144$ time-slots of 10 minutes. In the same way, each household $i$, has an aggregated power consumption profile which corresponds to the sum of the power usage of the individual appliances: $x_{i}=\sum_{a_{i}} x_{i, a_{i}}$, with $x_{i}=$ $\left(x_{i}(1), \ldots, x_{i}(T)\right)$, and $x_{i}(t)$ the aggregated power usage of household $i$ at time $t$, with $1 \leq t \leq T$.

We consider that appliance $a_{i}$ can evaluate how difficult is to realize a power profile $x_{i, a_{i}}$ through a function $f_{i, a_{i}}$ : $\mathbb{R}^{T} \rightarrow \mathbb{R}$, and that the appliance $a_{i}$ can be scheduled and controlled -with a given degree of uncertainty- through its associated control signal $u_{i, a_{i}} \in \mathcal{U}_{i, a_{i}}$. A control signal could indicate when to start using the appliance (e.g. in the case of a washing machine), when to start charging the appliance (e.g. an electric vehicle), or set the power usage level of the appliance (e.g. the intensity of the lighting or the air conditioner). This control should be selected such that it maximizes the QoL of the household $i$ but taking into account the uncertainties in achieving the profile $x_{i, a_{i}}$ :

$$
f_{i, a_{i}}\left(x_{i, a_{i}}\right)=\min _{u_{i, a_{i}} \in \mathcal{U}_{i, a_{i}}}\left[f_{i, a_{i}}^{u}\left(u_{i, a_{i}}\right)+f_{i, a_{i}}^{x \mid u}\left(x_{i, a_{i}}, u_{i, a_{i}}\right)\right] .
$$

The first term, $f_{i, a_{i}}^{u}\left(u_{i, a_{i}}\right)$, represents the dissatisfaction (degree of deviation from common pattern usage) associated with control $u_{i, a_{i}}$, and it can be understood as a measure of the flexibility in the appliance usage, or as a measure of the uncertainty of the control $u_{i, a_{i}}$ being applied. The second term, $f_{i, a_{i}}^{x \mid u}\left(x_{i, a_{i}}, u_{i, a_{i}}\right)$, represents the uncertainty of achieving the power usage profile $x_{i, a_{i}}$ for the control signal $u_{i, a_{i}}$, thus when a profile $x_{i, a_{i}}$ is not achievable by a given control signal $u_{i, a_{i}}$, the function $f_{i, a_{i}}^{x \mid u}\left(x_{i, a_{i}}, u_{i, a_{i}}\right)$ would give a large (possible infinite) value, thus also allowing to encode restrictions or uncertainties in achieving certain profiles. In Section III-F we present an implementation of such function $f_{i, a_{i}}\left(x_{i, a_{i}}\right)$ using a generative probabilistic model. We assume that the household $i$ has access to $f_{i, a_{i}}\left(x_{i, a_{i}}\right)$, and that it can schedule actions $u_{i, a_{i}}$ for the appliances.

The overall household's goal is to minimize the aggregated 
dissatisfaction and the uncertainty associated with the power usage profile of all appliances:

$$
\min _{\left\{x_{i, a_{i}}\right\}_{a_{i}}}\left[\sum_{a_{i}=1}^{N_{i}} f_{i, a_{i}}\left(x_{i, a_{i}}\right)\right] \text {. }
$$

It is important to recall that the control of the appliances will not be enforced: it is applied only when possible. For example, the charging of an electric vehicle can start only once the vehicle has been plugged, or the specific timing could be specified by the user. Thus for each time step $t$, there will be a possible different cost function for appliance $a_{i}$. We assume that the appliance (or its user) may inform the household's EMS, through the day, the usage timing of the appliance (e.g. when the rice cooker will be on), thus the household will have an updated $f_{i, a_{i}}^{t}\left(x_{i, a_{i}}\right)$ the moment this information is given. In general we will consider that the household may update the model at each time step $f_{i, a_{i}}^{t}\left(x_{i, a_{i}}\right)$, but we will omit the $t$ index unless necessary. Given that the cost function may change over time, and that the control may not be applied as planned, at each time step the households and the coordinator will negotiate a new power usage profile for the remainder of the day. This is expected to happen through the day, thus the control signals will be updated and applied at each time step $t$.

\section{B. Coordinator}

The objective of the community is to balance the aggregated power usage. This will be measured by a shared a global cost $g\left(\sum_{i=1}^{N} x_{i}\right)$, with $x_{i}=\sum_{a_{i}=1}^{N_{i}} x_{i, a_{i}}$, that depends on the aggregated power usage profile $\sum_{i=1}^{N} x_{i}$ (the power usage profile of the community). The coordinator's goal is to help find a good trade-off between the shared cost $g\left(\sum_{i=1}^{N} x_{i}\right)$, and the QoL of all households. This multiobjective optimization problem will be solved through the following single-objective optimization formulation:

$$
\min _{\left\{x_{i, a_{i}}\right\}_{i, a_{i}}}\left[\sum_{i=1}^{N} \sum_{a_{i}=1}^{N_{i}} f_{i, a_{i}}\left(x_{i, a_{i}}\right)+g\left(\sum_{i=1}^{N} \sum_{a_{i}=1}^{N_{i}} x_{i, a_{i}}\right)\right] .
$$

This last problem will be solved by the coordinator and the households by using a receding horizon formulation, through a distributed optimization, and will be implemented as an iterative negotiation between the coordinator and the households. The negotiation will take place several times during the day, as the households may deviate from their scheduled power usage profile.

In order to simplify the notation, in the following we will consider that each household consists of just one appliance, although the proposed solution still applies to the general case. Thus, in this simplified setting, the coordinator and the households solve the following problem:

$$
J=\min _{\left\{x_{i}\right\}_{i}}\left[\sum_{i=1}^{N} f_{i}\left(x_{i}\right)+g\left(\sum_{i=1}^{N} x_{i}\right)\right],
$$

where in the remainder of the paper $x_{i}$ represents a household with a single appliance, and $f_{i}\left(x_{i}\right)$ is the model of a single appliance.

\section{Receding horizon}

The problem formulated in Eq. (4) is solved with a receding horizon, using the following procedure: at each time step a new control policy is found for a given future time range, but that policy is used only in the next time step.

We write $x_{i}^{\left(t_{1}, t_{2}\right)}=\left(x_{i}\left(t_{1}\right), \ldots, x_{i}\left(t_{2}\right)\right) \in \mathbb{R}^{t_{2}-t_{1}+1}, 1 \leq$ $t_{1} \leq t_{2} \leq T$ to refer to a slice of the power profile $x_{i}$. Then, at each time step $t=1, \ldots T-1$, a new schedule is found for the time range $[t+1, T]$ by solving the following finite horizon problem:

$$
J_{t}=\min _{\left\{x_{i}^{(t+1, T)}\right\}_{i}}\left[\sum_{i=1}^{N} f_{i}^{t}\left(x_{i}\right)+g\left(\sum_{i=1}^{N} x_{i}\right)\right],
$$

with $x_{i}^{(t+1, T)}$ the planned power usage profiles of the $i$-th household, and $x_{i}=\left(x_{i}^{(1, t)}, x_{i}^{(t+1, T)}\right)$. Note that at time $t$ we can only aim to balance the future power profiles $x_{i}^{(t+1, T)}$, and that $f_{i}^{t}\left(x_{i}\right)$ is the model of household $i$ available at time $t$. The policy obtained from this minimization problem is then used at time step $t+1$, and this procedure is repeated for all $t=0, \ldots, T-1$. Note that $g(\cdot)$ could also change over time, though for simplicity we consider it is fixed.

Day-ahead schedule: At time step $t=0$, a the day-ahead schedule [11] is found for $J_{0}$, or equivalently:

$$
\left\{x_{i}^{(1, T)^{*}}\right\}_{i}=\underset{\left\{x_{i}^{(1, T)}\right\}_{i}}{\arg \min }\left[\sum_{i=1}^{N} f_{i}^{0}\left(x_{i}\right)+g^{0}\left(\sum_{i=1}^{N} x_{i}\right)\right],
$$

with $f_{i}^{0}\left(x_{i}\right)$ the day-ahead model of the household $i$ and $g^{0}\left(\sum_{i=1}^{N} x_{i}\right)$ the day-ahead global cost. The found solution can be used as an aggregated reference profile of the community for the rest of the day: $\sum_{i=1}^{N} r_{i}=\sum_{i=1}^{N} x_{i}^{(1, T)^{*}}$.

\section{Distributed Optimization}

As mentioned, at each time step $t$ we aim to solve Eq. (5). The goal is to solve this problem in a distributed way. For this, let $z_{i}$ be duplicate variables of $x_{i}$, thus:

$$
\begin{gathered}
J=\min _{\left\{x_{i}, z_{i}\right\}_{i}}\left[\sum_{i=1}^{N} f_{i}\left(x_{i}\right)+g\left(\sum_{i=1}^{N} z_{i}\right)\right] \\
\text { s.t } z_{i}=x_{i} \forall i,
\end{gathered}
$$

where we have omitted the time index $t$ for clarity. Letting $\nu_{i}=\frac{\lambda_{i}}{\rho}$ be the scaled Lagrangian multipliers, we get the corresponding augmented Lagrangian in scaled form of $J$ (refer to [25], [11] for details):

$$
\begin{aligned}
\tilde{J}= & \min _{\left\{x_{i}, z_{i}\right\}_{i}}\left[\sum_{i=1}^{N} f_{i}\left(x_{i}\right)+g\left(\sum_{i=1}^{N} z_{i}\right)\right. \\
& \left.+\frac{\rho}{2} \sum_{i=1}^{N}\left\|x_{i}-z_{i}+\nu_{i}\right\|^{2}-\frac{\rho}{2} \sum_{i=1}^{N}\left\|\nu_{i}\right\|^{2}\right] .
\end{aligned}
$$

Using this scaled form of the augmented Lagrangian, we now use the ADMM algorithm [25], which decomposes this optimization problem in a three step iterative procedure. In Eq. (7) there are individual costs $f_{i}\left(x_{i}\right)$ and a shared cost $g\left(\sum_{i} x_{i}\right)$, formulation that corresponds to a canonical 
problem known as the sharing problem. In this case, by applying the ADMM procedure, Eq. (8) is solved by the following three-step iterative procedure. At iteration $k$ :

$$
\begin{aligned}
x_{i}^{(k+1)} & :=\underset{x_{i}}{\arg \min }\left[f_{i}\left(x_{i}\right)+\frac{\rho}{2}\left\|x_{i}-z_{i}^{(k)}+\nu_{i}^{(k)}\right\|^{2}\right] \\
z_{i}^{(k+1)} & :=\underset{z}{\arg \min }\left[g\left(\sum_{i} z_{i}\right)+\frac{\rho}{2} \sum_{i}\left\|z_{i}-x_{i}^{(k+1)}-\nu_{i}^{(k)}\right\|^{2}\right] \\
\nu_{i}^{(k+1)} & :=\nu_{i}^{(k)}+x_{i}^{(k+1)}-z_{i}^{(k+1)} .
\end{aligned}
$$

By noting that we only need to use the average of $z_{i}, \bar{z}$, that $\nu_{i}^{(k)}=\nu^{(k)} \forall i$ (see [25] for details), and by defining $b^{(k)}=\bar{x}^{(k)}-\bar{z}^{(k)}+\nu^{(k)}$ (with $\bar{q}=\frac{1}{N} \sum_{i} q_{i}$ ), Eq. (9) can be rewritten as:

$$
\begin{aligned}
& \text { 1: } x_{i}^{(k+1)}:=\underset{x_{i}}{\arg \min }\left[f_{i}\left(x_{i}\right)+\frac{\rho}{2}\left\|x_{i}-x_{i}^{(k)}+b^{(k)}\right\|^{2}\right] \\
& \text { 2: } \bar{z}^{(k+1)}:=\underset{\bar{z}}{\arg \min }\left[g(N \bar{z})+\frac{N \rho}{2}\left\|\bar{z}-\bar{x}^{(k+1)}-\nu^{(k)}\right\|^{2}\right] \\
& \text { 3: } \nu^{(k+1)}:=\nu^{(k)}+\bar{x}^{(k+1)}-\bar{z}^{(k+1)},
\end{aligned}
$$

with $b^{(0)}=0, \nu^{(0)}=0$, and $x_{i}^{(0)}=\arg \min _{x_{i}}\left[f_{i}\left(x_{i}\right)\right]$.

\section{E. Negotiation protocol}

The receding horizon optimization problem stated in Eq. (5), and formulated as Eq. (7), is solved at each time step $t$ following the iterative procedure in Eq. (10). In this iterative process, each household solves the step 1 associated to its own power profile, while the the coordinator solves the step two and three. This iterative process can be seen as negotiation, where at iteration $k$ :

First, each household $i=1, \ldots, N$

- obtains its power usage profile $x_{i}^{(k+1)}$ using the current broadcast signal $b^{(k)}$ (step 1, Eq. (10)).

\section{Second, the coordinator}

- collects the profiles $x_{i}^{(k+1)}, i=1, \ldots, N$,

- averages the profiles $\bar{x}^{(k+1)}=\frac{1}{N} \sum_{i} x_{i}^{(k+1)}$,

- calculates $\bar{z}^{k+1}$ (step 2, Eq. (10)),

- updates the dual variables $\nu^{(k+1)}$ (step 3, Eq. (10)), and

- broadcasts $b^{(k+1)}$ to all households.

This negotiation goes on until the number of maximum iterations, $K$, is achieved, or until the stopping criteria is achieved (bounds in the primal and dual residuals [25]). This algorithm is synchronous at each iteration, with the coordinator waiting for the profiles of all households, and the households waiting to receive the broadcast signal from the coordination to generate new power profiles.

There are two important things to note. First, only the coordinator has access to the power usage profile of the households (households do not have access to each other profiles). This has the advantage of reducing the required communication bandwidth, and also gives more privacy to the users. In addition, the coordinator has access only to the profile of each household, but it does not have access to the individual cost functions, nor it can control the appliances.
Secondly, the coordinator communicates the same broadcast signal to all households, thus reducing the communication bandwidth, but also giving symmetric information to all households.

\section{F. Generative probabilistic model of the appliances}

The control of the appliances is done by the household, which has access to the appliance model $f_{i}^{t}\left(x_{i}\right)$ at time $t$. In the following we will omit the time subscript $t$ for clarity. We recall that the appliance model is:

$$
f_{i}\left(x_{i}\right)=\min _{u_{i}}\left[f_{i}^{u}\left(u_{i}\right)+f_{i}^{x \mid u}\left(x_{i}, u_{i}\right)\right] .
$$

We give a particular implementation that can be used to model different kinds of appliances by following [11]. We define a generative model $P\left(x_{i}, u_{i}\right)=P\left(u_{i}\right) P\left(x_{i} \mid u_{i}\right)$, with $P\left(u_{i}\right)$ a measure of how natural is control $u_{i}$, and $P\left(x_{i} \mid u_{i}\right)$ a measure of the certainty of achieving a given power usage profile given a control signal $u_{i}$. Therefore we define:

$$
\begin{aligned}
f_{i}^{u}\left(u_{i}\right) & \doteq-\log P\left(u_{i}\right), \\
f_{i}^{x \mid u}\left(x_{i}, u_{i}\right) & \doteq-\log P\left(x_{i} \mid u_{i}\right), \\
f_{i}\left(x_{i}\right) & \doteq \min _{u_{i} \in \mathcal{U}_{i}}\left[-\log P\left(x_{i}, u_{i}\right)\right] .
\end{aligned}
$$

In the cases when $P\left(x_{i}, u_{i}\right)$ becomes zero, $f_{i}\left(x_{i}\right)$ becomes $+\infty$, indicating that the associated profile $x_{i}$ is not achievable. A particular instance of this is when $P\left(x_{i} \mid u_{i}\right)$ is given by the delta function $\delta\left(x_{i}-\chi_{i}\left(u_{i}\right)\right)$, with $\chi_{i}: \mathcal{U}_{i} \rightarrow \mathbb{R}^{T}$.

A key aspect in the balance of the power profiles is to schedule the power usage of the households and appliances. In many cases, the usage timing is more important (and also more controllable) than the power level used. Thus, in this work we focus on the usage timing of the appliances. For this, we model the probability of the control $u_{i}, P\left(u_{i}\right)$, using a hidden semi-Markov model (HSMM) [34][35]. This method explicitly models time-varying signals as a sequence of time intervals (or modes) and is popular in the speech recognition community.

Following HSMM, we model the profile $x_{i}$ by a sequence of discrete states $s_{i, t} \in \mathcal{Q}_{i} \doteq\left\{q_{i, m}\right\}_{m=1, \ldots, M_{i}}$, where each of these states, $s_{i, t}$, represents the state of appliance $i$ at time $t$ (so called modes). We assume that the control variables $u_{i}$ are these modes at time $t=1, \ldots, T$. For clarity, in the following we will omit the $i$ index and we will use $y_{t}$ instead of $x_{i, t}$ to avoid the abuse of symbol $x$. Thus $x_{i}=$ $\left(y_{1}, \ldots, y_{T}\right)=y_{1: T}$. In a HSMM, the output at time $t, y_{t}$, is assumed to be determined only by $s_{t}$, thus:

$$
P\left(x_{i} \mid u_{i}\right)=P\left(y_{1: T} \mid s_{1: T}\right)=\prod_{t=1}^{T} P\left(y_{t} \mid s_{t}\right),
$$

where $P\left(y_{t} \mid s_{t}\right)$ is the output probability distribution.

On the other hand, $P\left(u_{i}\right)=P\left(s_{1: T}\right)$ is modeled as a segment-based process. Following [36], let $\tau_{t} \geq 1$ be a random variable denoting the remaining time at the current mode $s_{t}$. Assume that the pair $\left(s_{t}, \tau_{t}\right)$ takes the value $\left(q_{m}, d\right)$, i.e. the mode at time $t$ current time is $q_{m}$ and the remaining time is $d$, then: 
- If $d>1$, then $\left(s_{t+1}, \tau_{t+1}\right)=\left(q_{m}, d-1\right)$ (no mode transition occurs), otherwise

- If $d=1$, then $\left(s_{t+1}, \tau_{t+1}\right)=\left(q_{m^{\prime}}, d^{\prime}\right)$ (mode transition). This process is modeled by a mode transition probability $P\left(\tau_{t}=d \mid s_{t}, \tau_{t-1}=1\right)$. The parameters of a HSMM are:

- Initial mode: $\pi_{m} \doteq P\left(s_{1}=q_{m}\right)$,

- Mode transition: $a_{m n} \doteq P\left(s_{t+1}=q_{n} \mid s_{t}=q_{m}, \tau=1\right)$,

- Duration: $p_{m}(d) \doteq P\left(\tau_{t}=d \mid s_{t}=q_{m}, \tau_{t-1}=1\right)$,

- Output: $b_{m}\left(y_{t}\right) \doteq P\left(y_{t} \mid s_{t}=q_{m}\right)$,

where $q_{m}, q_{n} \in \mathcal{Q}, d \geq 1$ and $y_{t} \in \mathbb{R}$. These parameters can be estimated using methods such as the EM-algorithm [36]. Given that our aim at this point is to show the effectiveness of the proposed distributed on-line coordination framework, in Section IV we will present simulation results where these parameters are not learned, but manually determined.

\section{G. Optimal mode scheduling}

The step 1 of the distributed optimization procedure described in Eq. (10) requires to solve a minimization problem that takes into account the described probabilistic generative model. The problem to be solved corresponds to:

$$
x_{i}^{(k+1)}=\underset{x_{i}}{\arg \min }\left[f_{i}\left(x_{i}\right)+\frac{\rho}{2}\left\|x_{i}-x_{i}^{(k)}+b^{(k)}\right\|^{2}\right] .
$$

If we note $F_{i}^{(k)}\left(x_{i}\right) \doteq \frac{\rho}{2}\left\|x_{i}-x_{i}^{(k)}+b^{(k)}\right\|^{2}$ and include the generative model, this is equivalent to solve:

$$
\begin{aligned}
& \underset{u_{i}}{\arg \min }\left[-\log P\left(u_{i}\right)+\min _{x_{i}}\left(-\log P\left(x_{i} \mid u_{i}\right)+F_{i}^{(k)}\left(x_{i}\right)\right)\right] \\
& =-\underset{u_{i}}{\arg \max }\left[\log P\left(u_{i}\right)+\max _{x_{i}}\left(\log P\left(x_{i} \mid u_{i}\right)-F_{i}^{(k)}\left(x_{i}\right)\right)\right] .
\end{aligned}
$$

Solving this directly requires evaluating $M^{T}$ sequences, which may be prohibitive for large numbers of modes, $M$, and time slots, $T$. However we can note that

$$
\begin{aligned}
& \max _{x_{i}}\left[\log P\left(x_{i} \mid u_{i}\right)-F_{i}^{(k)}\left(x_{i}\right)\right] \\
= & \max _{y_{1: T}}\left[\sum_{t=1}^{T} \log P\left(y_{t} \mid s_{t}\right)-F_{i, t}^{(k)}\left(y_{t}\right)\right] .
\end{aligned}
$$

with $F_{i, t}^{(k)}\left(y_{t}\right)$ the $t$-th component of $F_{i}^{(k)}\left(x_{i}\right)$. Then we can define $G_{t}\left(s_{t}\right) \doteq \max _{y_{t}}\left(\log P\left(y_{t} \mid s_{t}\right)-F_{i, t}^{(k)}\left(x_{i}\right)\right)$, thus

$$
\max _{y_{1: T}}\left[\sum_{t=1}^{T} \log P\left(y_{t} \mid s_{t}\right)-F_{i, t}^{(k)}\left(y_{t}\right)\right]=\sum_{t=1}^{T} G_{t}\left(s_{t}\right) .
$$

Using this formulation, Eq. (15) can be solved efficiently by using dynamic programming. In addition [11]:

$$
\begin{array}{r}
P\left(s_{t}=q_{m}, \tau_{t}=d\right)=P\left(s_{t-1}=q_{m}, \tau_{t-1}=d+1\right) \\
+P\left(\tau_{t-1}=1, s_{t}=q_{m}\right) P\left(\tau_{t}=d \mid \tau_{t-1}=1, s_{t}=q_{m}\right)
\end{array}
$$

and

$$
P\left(\tau_{t-1}=1, s_{t}=q_{m}\right)=\sum_{n \neq m} a_{n m} P\left(\tau_{t-1}=1, s_{t-1}=q_{n}\right),
$$

thus, to arrive at $\left(s_{t}, \tau_{t}\right)=\left(q_{m}, d\right)$ from $s_{t-1}$ there are only $M$ paths depending on $s_{t-1}$. Thus, by defining $\Phi_{t}(m, d)$ as:

$$
\max _{s_{1: t-1}}\left[\log P\left(s_{1: t-1}, s_{t}=q_{m}, \tau_{t}=d\right)+\sum_{t^{\prime}=1}^{t-1} G_{t^{\prime}}\left(s_{t^{\prime}}\right)\right]
$$

we can write the following recursive formulation:

$$
\begin{aligned}
& \Phi_{t}(m, d)=\max \left[\Phi_{t-1}(m, d+1)+G_{t-1}\left(q_{m}\right),\right. \\
& \left.\log p_{m}(d)+\max _{n}\left(\Phi_{t-1}(n, 1)+\log a_{n m}+G_{t-1}\left(q_{n}\right)\right)\right],
\end{aligned}
$$

with the maximization done over all possible $s_{t-1}$. From the definition, the initialization is given by

$\Phi_{1}(m, d)=\log P\left(s_{1}=q_{m}, \tau_{1}=d\right)=\log p_{m}(d)+\log \pi_{m}$.

The solution of Eq. (15) is given by $\max _{m, d}\left(\Phi_{T}(m, d)+\right.$ $\left.G_{T}\left(q_{m}\right)\right)$ and the corresponding mode scheduling is obtained by tracing back the optimal path of the recursion. Thus, this gives the control signal and the associated power profile of step 1 of the distributed scheduling in Eq. (9).

\section{H. Appliance model update and adjustment}

Similarly to [24] we consider that the available information regarding the appliances may change during the day. In our formulation, at time $t$, this information is encoded in the generative model $f_{i}^{t}\left(x_{i}\right)$. A simple example of a change in $f_{i}^{t}\left(x_{i}\right)$, consists in the case when the control $u_{i}=\hat{u}_{i}$ becomes fixed by the appliance, and that the control is informed to the household at time $t$ (e.g. a rice cooker with a timer). Then, the probabilities in the generative model at time $t$ becomes $P^{t}\left(u_{i}=\hat{u}_{i}\right)=1$ and $P^{t}\left(u_{i} \neq \hat{u}_{i}\right)=0$, and therefore $f_{i}^{t}\left(x_{i}\right)$ is minimized at $f_{i}^{t}\left(x_{i}\right)=0$ for $u_{i}=\hat{u}_{i}$ (but becomes $f_{i}^{t}\left(x_{i}\right)=\infty$ for $\left.u_{i} \neq \hat{u}_{i}\right)$. In the ideal case, the schedule should be informed to the household as soon as fixed, so that the on-line negotiation can take this information into account and a new schedule can be planed to adjust in advance, instead of being rescheduled in a reactive way.

Also note that $f_{i}^{t}\left(x_{i}\right)$ must be adjusted using past observations of the power profile, that is, the observed values until time $t, x_{i}^{(1, t)}$. In particular, the duration probabilities must be adjusted over time. For example, if at time $t$ the household $i$ has not yet started to be used, the duration probability of the first mode (start time) will be zero for all values smaller or equal than $t$. Basically, the probability distribution of the duration becomes truncated for small values of $d$ (earlier modes) and for large values of $d$ (later modes).

\section{Simulation Results}

We evaluate the effectiveness of the proposed framework in a simulated scenario (similar to [11]) designed to analyze the effect of two factors related to the on-line scheduling: i) how well the control handles the inflexibility of a group households, and ii) how well a notification in advance of the actual self-defined schedule of inflexible households, improves the coordinated schedule.

The scenario consists of a group of $N=20$ households, each having a single appliance that corresponds to an 
electric vehicle that must be charged ( $3 \mathrm{kWh})$ continuously for about $3 \mathrm{hrs}$ during the day. We divided a day into $T=144$, 10-minute slots, thus the charging takes about 18 time-slots. The community tries to minimize its deviation from a reference aggregated power usage during the day: $g\left(\sum_{i} x_{i}\right)=\left\|\sum_{i} x_{i}-r\right\|^{2}$, with $r=\sum_{i} r_{i}$ the day-ahead aggregated reference power profile obtained following Eq. (6). Note that is the community the one that tries to follow the aggregated reference profile $r$ and not each household trying to follow its own reference profile $r_{i}$. In the experiments, the day-ahead schedule is obtained at time $t=0$ for $g^{0}\left(\sum_{i} x_{i}\right)=\left\|\sum_{i} x_{i}\right\|^{2}$, i.e. the day-ahead schedule seeks to balance the aggregated power profile.

Each electric vehicle is represented by three modes $(M=$ 3): $\mathcal{Q}=\left\{q_{1}, q_{2}, q_{3}\right\}$, with $q_{1}$ representing the period before charging the electric car, $q_{2}$ the charging period, and $q_{3}$ the period of after the charging (the remaining of the day). In other words, we assume that the initial mode and the transition probabilities are identical for all the household, with a deterministic transition pattern $q_{1} \rightarrow q_{2} \rightarrow q_{3}$. In addition, as we want to focus on the mode scheduling aspect, we consider an output distribution given by $P\left(y_{t} \mid s_{t}\right)=1_{\left[y_{t}=\mathcal{X}\left(s_{t}\right)\right]}$, with $1_{[\circ]}$ the indicator function and $\mathcal{X}\left(s_{t}\right)=0,1000,0[W]$ for $s_{t}=q_{1}, q_{2}, q_{3}$ respectively. We assume that each appliance can present one of the following two behaviors:

Flexible appliance: It follows the coordinated schedule and it has a fixed model through the day:

$$
f_{i}^{t}\left(x_{i}\right)=f_{i}^{0}\left(x_{i}\right) \forall t
$$

Inflexible appliance: It does not follow the scheduled control, and it will inform the actual one at time $t_{i}^{\prime}>0$ :

$$
f_{i}^{t}\left(x_{i}\right)= \begin{cases}f_{i}^{0}\left(x_{i}\right), & \text { if } t<t_{i}^{\prime} \\ f_{i}^{\prime}\left(x_{i}\right), & \text { if } t \geq t_{i}^{\prime}\end{cases}
$$

where $f_{i}^{\prime}\left(x_{i}\right)$ is the cost function used by the appliances from time $t_{i}^{\prime}$. An inflexible appliance can not be controlled: it has fixed duration times for the 3 modes $\left(q_{1}, q_{2}, q_{3}\right)$, with the second mode (charging) starting at time $\tilde{t}_{i}$.

In the experiments a percentage $(p=15 \%, 30 \%, 45 \%)$ of households are flexible (out of the $N$ ), and the coordinator does not know in advance neither $p$ nor which households are inflexible. An inflexible appliance informs its self-defined schedule at time $t_{i}^{\prime}=\tilde{t}_{i}-\delta_{i}$, where $\tilde{t}_{i}$ is the actual starting time (duration of mode 1), and where $\delta_{i} \in \mathbb{R}$ indicates how early in advance the self-defined scheduled will be informed to the household (relative to the usage time $\tilde{t}_{i}$ ). Thus, the larger the value of $\delta_{i}$, the longer the time the community can to adjust, in advance, to unplanned schedule changes. A $\delta_{i} \leq 0$ means the appliance does not inform anything to the household: it just starts charging the vehicle at time $\tilde{t}_{i}$ and the household does not know it in advance. For the simulations we consider that $\delta_{i}$ follows a Gaussian distribution with mean $m_{\delta} \in\{0,10,20,30\}$ and standard deviation equal $\sigma_{\delta}=18$, while $\tilde{t}_{i}$ is sampled from the same distribution as the duration of mode $q_{1}$ of the appliance (defined below).

In the day-ahead model, the duration of mode $m$ follows a Gaussian distribution $\mathcal{N}\left(\mu_{m}, \sigma_{m}^{2}\right)$, having the following parameters ( $\mu_{1}$ and $\mu_{3}$ are different for every user):

- $\mu_{1}$ is uniformly distributed in $[50,55] ; \sigma_{1}=10$.

- $\mu_{2}=18$ (3 hrs); $\sigma_{2}=1$.

- $\mu_{3}=T-\left(\mu_{1}+\mu_{2}\right) ; \sigma_{3}=10$.

The simulation was ran 10 times, each time using different samples from the corresponding distributions. The obtained results are summarized in Figure 3, while Figure 2 presents results for a particular run for four methods:

- Without coordination (dashed black)

- Reference profile $r=\sum_{i} r_{i}$ (dot-dashed green)

- Without on-line coordination (filled blue)

- With on-line coordination (solid red) (proposed)

The results of with (red) / without (blue) on-line coordination are analyzed (Figure 3 ) using three measures (smaller values are better), where $x_{i}$ represents the case with (without) online coordination:

Power balance: ratio of the $\ell_{2}$-norm of the aggregated power without coordination $\left(\tilde{x}_{i}\right)$ and the $\ell_{2}$-norm of the aggregated power: $\left\|\sum_{i} \tilde{x}_{i}\right\|^{2} /\left\|\sum_{i} x_{i}\right\|^{2}$.

Peak Shaving: ratio of the maximum of the aggregated power power without coordination $\left(\tilde{x}_{i}\right)$ and the maximum of the aggregated power: $\max _{t}\left(\sum_{i} \tilde{x}_{i}(t)\right) / \max _{t}\left(\sum_{i} x_{i}(t)\right)$.

Deviation from reference: $\ell_{2}$-norm of the difference of the aggregated power and the reference profile, normalized by the $\ell_{2}$-norm of the reference: $\left\|\sum_{i} x_{i}-r\right\|^{2} /\|r\|^{2}$.

From the results it can be observed that: $i$ ) The day-ahead control policy can balance the power, reducing both the $\ell_{2}$ norm and the maximum peak, even when only $p=15 \%$ households are flexible $\left(13.7 \% \ell_{2}\right.$-norm reduction and $9 \%$ peak reduction). ii) The on-line control can further improve the power balance, further reducing the $\ell_{2}$-norm and the maximum peak. When $\delta$ is small (e.g. $\delta=0$, Figure 2, first column) the control is basically reactive, postponing the schedule and thus producing a small peak rebound, but nevertheless flattening power usage. When $\delta$ is large (e.g. $\delta=30$, Figure 2, fourth column) the control is not reactive, but reschedules the charging of more electric vehicles before the main peak, producing no peak rebounds (achieving a $17.2 \%$ $\ell_{2}$-norm reduction, a $15.5 \%$ peak reduction). iii) When $p=$ $45 \%$ (Figure 3, third column) and the inflexible households notify their schedule well in advance $(\delta=30)$, the reduction in $\ell_{2}$-norm and maximum peak (first and second row) are rather large (35\% and $45 \%$ respectively), also achieving an aggregated profile (third row) that is much closer to the reference one (45\% normalized difference from reference).

\section{CONCLUSiON}

In the current paper we propose a framework for distributed on-line power demand balancing of households communities. The framework is designed such that a community of household coordinates and updates the schedule of their appliances power usage, instead of the appliances being controlled by the utility company. The goal is to balance the aggregated power of the community of households taking into account the Quality of Life (QoL) of the users. This 

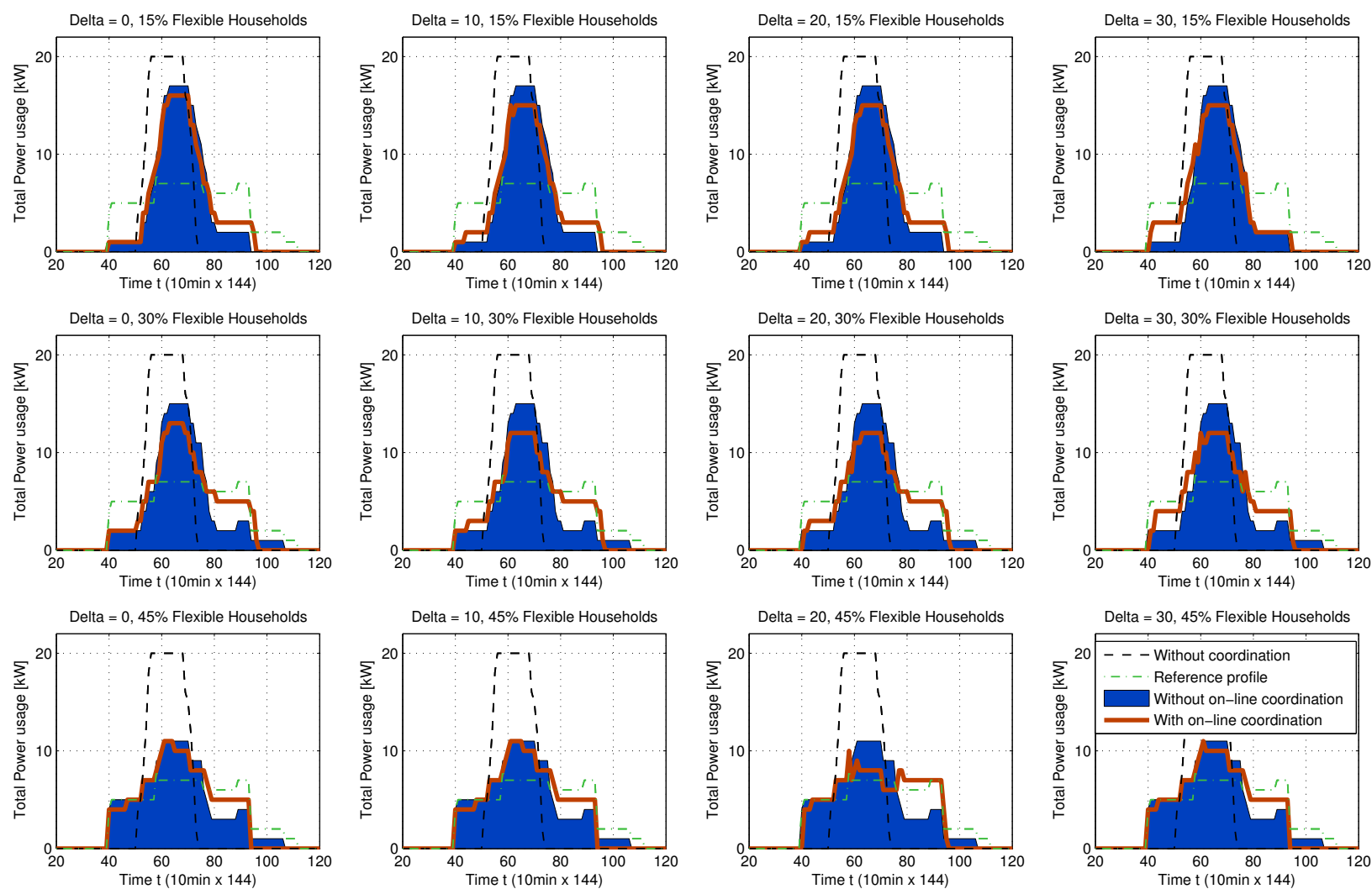

Fig. 2. Aggregated power usage of 20 electric vehicles during one day. Results for (rows) different number of flexible households ( $p=15,30$ and $45 \%$ ), and (columns) different distributions of notification time (Gaussian parametrized by mean $\delta \in\{0,10,20,30\}$ and $\sigma_{\delta}=18$ ) of inflexible households.
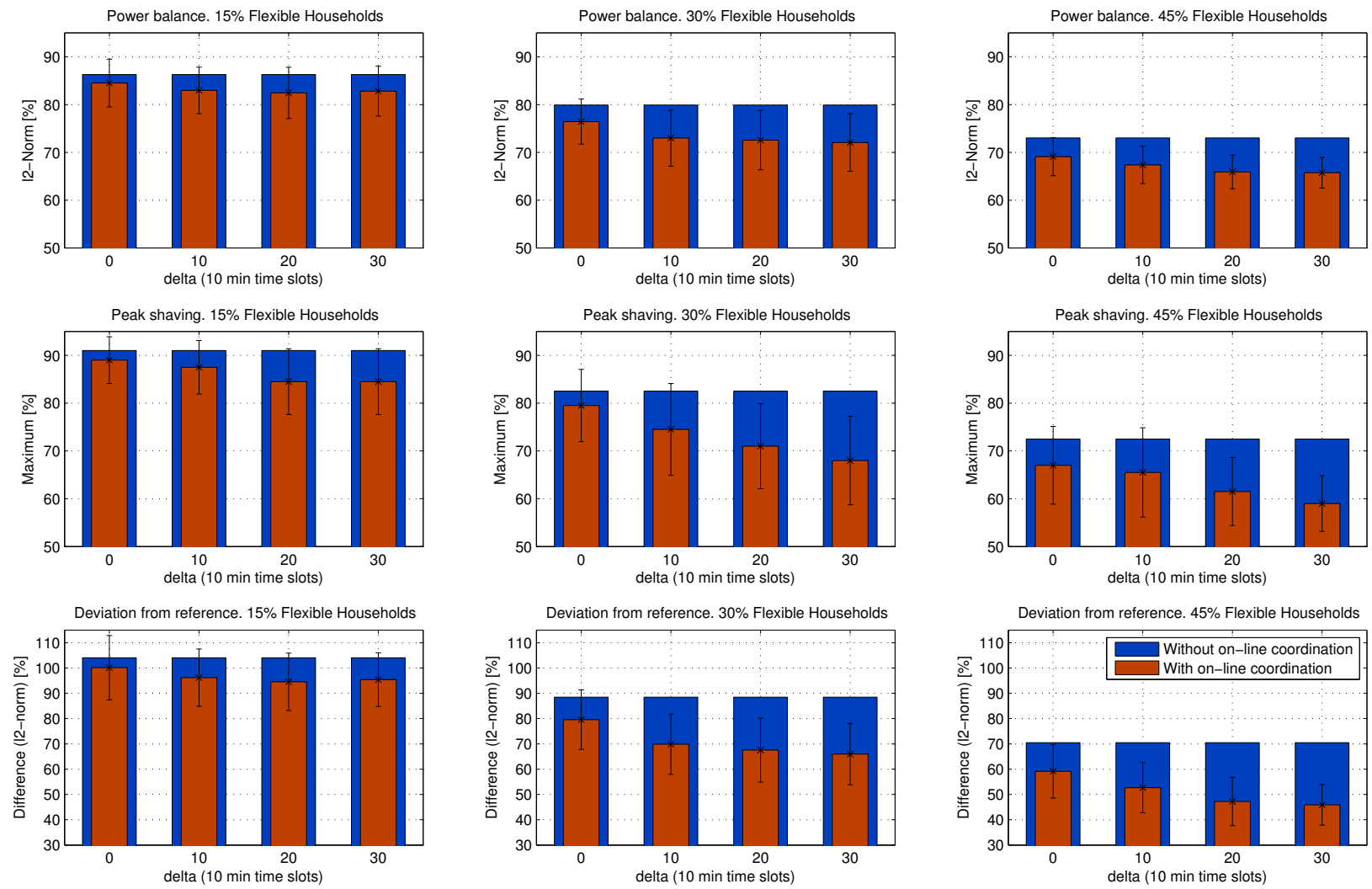

Fig. 3. Average results over 10 simulation runs. Results are indicated as percentages relative to the case when no control is applied (power balance, first row; peak shaving, second row) and to the reference profile (deviation from reference, third row). Lower values are better. Results for (columns) different number of flexible households $(p=15,30$ and $45 \%)$. The x-axis indicates the distribution of the notification time of inflexible households (Gaussian parametrized by mean $\delta \in\{0,10,20,30\}$ and $\left.\sigma_{\delta}=18\right)$. 
is formulated as a receding horizon optimization problem where we seek not to deviate much from a reference aggregated usage profile and to minimize deviations from common usage patterns. For this, a generative probabilistic model of appliance power usage is considered to determine the optimal coordinated schedule of the appliances. We analyze the case where the households are not enforced to follow the coordinated schedule (when some of them do not, they will negotiate a new schedule for the next time step), and where the appliances may notify in advance their self-defined schedule, allowing to update the generative model with actual usage information, and thus improve the power balancing and reduce peak rebounds. As future work, and similarly to the analysis done in [37] for the decentralized consensus problem, we plan to analyze the proposed framework when the objective functions change over time.

\section{REFERENCES}

[1] P. Palensky and D. Dietrich, "Demand side management: Demand response, intelligent energy systems, and smart loads," IEEE Transactions on Industrial Informatics, vol. 7, no. 3, pp. 381-388, 2011.

[2] J. Aghaei and M. Alizadeh, "Demand response in smart electricity grids equipped with renewable energy sources: A review," Renewable and Sustainable Energy Reviews, vol. 18, 2013.

[3] M. N. Ullah, A. Mahmood, S. Razzaq, M. Ilahi, R. D. Khan, and N. Javaid, "A survey of different residential energy consumption controlling techniques for autonomous dsm in future smart grid communications," CoRR, vol. abs/1306.1134, 2013.

[4] M. H. Albadi and E. El-Saadany, "Demand response in electricity markets: An overview," in Power Engineering Society General Meeting, 2007. IEEE, 2007, pp. 1-5.

[5] M. Kefayati and R. Baldick, "On energy delivery to delay-averse flexible loads: Optimal algorithm, consumer value and network level impacts," in Decision and Control (CDC), 2012 IEEE 51st Annual Conference on, 2012, pp. 2401-2408.

[6] S. Caron and G. Kesidis, "Incentive-Based energy consumption scheduling algorithms for the smart grid," in Smart Grid Communications (SmartGridComm), 2010 First IEEE International Conference on, 2010, pp. 391-396.

[7] A. Schulke, J. Bauknecht, and J. Haussler, "Power demand shifting with smart consumers: A platform for power grid friendly consumption control strategies," in Smart Grid Communications (SmartGridComm), 2010 First IEEE International Conference on, 2010, pp. 437-442.

[8] T. Kato, K. Yuasa, and T. Matsuyama, "Energy on demand: Efficient and versatile energy control system for home energy management," in Smart Grid Communications (SmartGridComm), 2011 IEEE International Conference on, 2011, pp. 392-397.

[9] P. Shenoy, "Demand-side load management in smart homes," in Communication Systems and Networks (COMSNETS), 2012 Fourth International Conference on, 2012.

[10] D. L. Ha, F. de Lamotte, and Q.-H. Huynh, "Real-time dynamic multilevel optimization for demand-side load management," in Industrial Engineering and Engineering Management, 2007 IEEE International Conference on, 2007, pp. 945-949.

[11] H. Kawashima, T. Kato, and T. Matsuyama, "Distributed mode scheduling for coordinated power balancing," in Smart Grid Communications (SmartGridComm), 2013 IEEE International Conference on, Oct 2013, pp. 19-24.

[12] A. Subramanian, M. Garcia, A. Dominguez-Garcia, D. Callaway, K. Poolla, and P. Varaiya, "Real-time scheduling of deferrable electric loads," in American Control Conference (ACC), 2012, pp. 3643-3650.

[13] M. Gonzalez Vaya and G. Andersson, "Centralized and decentralized approaches to smart charging of plug-in vehicles," in Power and Energy Society General Meeting, 2012 IEEE, 2012, pp. 1-8.

[14] J. Mathieu, S. Koch, and D. Callaway, "State estimation and control of electric loads to manage real-time energy imbalance," Power Systems, IEEE Transactions on, vol. 28, no. 1, pp. 430-440, 2013.

[15] Z. Ma, D. Callaway, and I. Hiskens, "Decentralized charging control for large populations of plug-in electric vehicles," in Decision and Control (CDC), 2010 49th IEEE Conference on, 2010, pp. 206-212.
[16] Z. Wang and S. Wang, "Grid power peak shaving and valley filling using Vehicle-to-Grid systems," IEEE Transactions on Power Delivery, vol. $28,2013$.

[17] E. Sortomme and E. Mohamed, "Optimal scheduling of Vehicle-toGrid energy and ancillary services," IEEE Transactions on Smart Grid, vol. 3, no. 1, pp. 351-359, 2012.

[18] S. Cady, A. Dominguez-Garcfa, and C. Hadjicostis, "Robust implementation of distributed algorithms for control of distributed energy resources," in North American Power Symp. (NAPS), 2011, pp. 1-5.

[19] M. Kraning, E. Chu, J. Lavaei, and S. Boyd, "Dynamic network energy management via proximal message passing," Optimization, vol. 1, no. 2, pp. 1-54, 2013.

[20] E. Wei and A. Ozdaglar, "Distributed alternating direction method of multipliers," in Decision and Control (CDC), 2012 IEEE 51 st Annual Conference on, 2012, pp. 5445-5450.

[21] M. Alizadeh, T.-H. Chang, and A. Scaglione, "Grid integration of distributed renewables through coordinated demand response," in Decision and Control (CDC), 2012 IEEE 51st Annual Conference on, 2012, pp. 3666-3671.

[22] M. Petersen, K. Edlund, L. Hansen, J. Bendtsen, and J. Stoustrup, "A taxonomy for modeling flexibility and a computationally efficient algorithm for dispatch in smart grids," in American Control Conference (ACC), 2013, 2013, pp. 1150-1156.

[23] S. Chen, P. Sinha, and N. Shroff, "Scheduling heterogeneous delay tolerant tasks in smart grid with renewable energy," in Decision and Control (CDC), IEEE 51st Annual Conf. on, 2012, pp. 1130-1135.

[24] P. Samadi, H. Mohsenian-Rad, V. Wong, and R. Schober, "Tackling the load uncertainty challenges for energy consumption scheduling in smart grid," Smart Grid, IEEE Transactions on, vol. 4, no. 2, pp. 1007-1016, 2013.

[25] S. Boyd, N. Parikh, E. Chu, B. Peleato, and J. Eckstein, "Distributed optimization and statistical learning via the alternating direction method of multipliers," Found. Trends Mach. Learn., vol. 3, no. 1, pp. 1-122, Jan. 2011.

[26] F. Lin, M. Fardad, and M. R. Jovanovic, "Algorithms for leader selection in large dynamical networks: Noise-corrupted leaders," in CDC-ECE. IEEE, 2011, pp. 2932-2937.

[27] C. Conte, T. summers, M. Zeilinger, M. Morari, and C. Jones, "Computational aspects of distributed optimization in model predictive control," in Decision and Control (CDC), 2012 IEEE 51st Annual Conference on, 2012, pp. 6819-6824.

[28] F. Dorfler, M. R. Jovanovic, M. Chertkov, and F. Bullo, "Sparse and optimal wide-area damping control in power networks," in American Control Conference (ACC), 2013, 2013, pp. 4289-4294.

[29] J. F. C. Mota, J. M. F. Xavier, P. M. Q. Aguiar, and M. Puschel, "Distributed ADMM for model predictive control and congestion control," in Decision and Control (CDC), 2012 IEEE 51st Annual Conference on. IEEE, 2012, pp. 5110-5115.

[30] J. Rivera, P. Wolfrum, S. Hirche, C. Goebel, and H.-A. Jacobsen, "Alternating direction method of multipliers for decentralized electric vehicle charging control," in Decision and Control (CDC), 2013 IEEE 52nd Annual Conference on, Dec 2013, pp. 6960-6965.

[31] A. Mercurio, A. D. Giorgio, and F. Purificato, "Optimal fully electric vehicle load balancing with an admm algorithm in smartgrids," in Control Automation (MED), 2013 21st Mediterranean Conference on, June 2013, pp. 119-124.

[32] T. Erseghe, "Distributed optimal power flow using admm," Power Systems, IEEE Transactions on, vol. PP, no. 99, pp. 1-11, 2014.

[33] A. Sun, D. Phan, and S. Ghosh, "Fully decentralized ac optimal power flow algorithms," in Power and Energy Society General Meeting (PES), 2013 IEEE, July 2013, pp. 1-5.

[34] M. Ostendorf, V. Digalakis, and O. A. Kimball, "From hmms to segment models: A unified view of stochastic modeling for speech recognition," IEEE Transactions on Speech and Audio Processing, vol. 4, pp. 360-378, 1995.

[35] S. Levinson, "Continuously variable duration hidden markov models for automatic speech recognition," Computer Speech and Language, vol. 1, no. 1, pp. $29-45,1986$.

[36] S.-Z. Yu and H. Kobayashi, "Practical implementation of an efficient forward-backward algorithm for an explicit-duration hidden markov model," Signal Processing, IEEE Transactions on, vol. 54, no. 5, pp. 1947-1951, 2006.

[37] Q. Ling and A. Ribeiro, "Decentralized dynamic optimization through the alternating direction method of multipliers," Signal Processing, IEEE Transactions on, vol. 62, no. 5, pp. 1185-1197, March 2014. 